\title{
Signaling pathways of nucleic acids for bone healing: A review
}

\begin{abstract}
Different kinds of nucleic acid (NA) molecules are promising therapeutic tools in a variety of tissues and their pathogenesis, and bone pathologies are not the exception. NAs interact in cytosol or nucleus to generate a specific response. Some of these NAs can be used to generate a positive response in relation to bone formation and differentiation in osteoblast and osteocyte. This work aims to briefly and clearly show main signaling pathways in osteoblasts and osteocytes, and to state the mechanism of how miRNA agonists (miRNA) and miRNA antagonists (antagomir) affect them. Thus, this summarizes the mechanism of promising therapeutic strategies for bone repair. NAs are fragile and can be degraded quickly outside the cells. These problems are more and more frequently resolved by nanotechnology and tissue engineering approaches. Further research in this field wills probably generate safe and therapeutic effective therapy in relation to bone healing.
\end{abstract}

Keywords: bone repair, miRNA, antagomir, osterix, runx2, signaling pathway, wnt
Volume 8 Issue 5 - 2020

\author{
Iván Nadir Camal Ruggieri,' Sara Feldman, ${ }^{1,2}$ \\ 'School of Medicine, LABOATEM - Osteoarticular Biology, \\ Tissue Engineering and Emerging Therapies Laboratory, \\ Biological Chemistry Cat., School of Medicine, Rosario National \\ University, Argentina \\ ${ }^{2}$ Research Council of theRosario National University, (CIUNR) \\ and CONICET, Argentina
}

Correspondence: Iván Nadir Camal Ruggieri, LABOATEM,

Santa Fe 3100, Rosario, Santa Fe, Argentina, Tel +54934I6507995,

Email ivannadircamalruggieri@gmail.com

Received:September 16, 2020 | Published: October 02, 2020
Abbreviations: NA, nucleic acid; siRNA, small interfering RNA; miRNA, microRNA analog; antagomir, microRNA antagonist; mRNA, messenger RNA; Fz, Frizzled; LRP5/6, low-density lipoprotein receptor-related protein 5 or 6 ; ROR, RAR-related orphan receptor; APC, activated protein C; GSK3, glycogen synthase kinase 3; CK1, casein kinase; TCF/LEF, T cell factor/lymphoid enhancer factor 1; OCN, osteocalcin; OPN, osteopontin; RANK-L, receptor activator for nuclear factor $\mathrm{\kappa B}$ ligand; ALP, alkaline phosphatase; OPG, osteoprotegerin; Col1, type 1 collagen; MMP-13, matrix metallopeptidase 13; PCP, planar cell polarity; YAP-TAZ, yesassociated protein - transcriptional co-activator with PDZ-binding motif; Wnt3a, Wnt family member 3a; DKK-1, dikkopf-related protein 1; SFRP-1, secreted Frizzled-related protein 1; Wnt5a, Wnt family member $5 \mathrm{a}$; Wnt5b, Wnt family member $5 \mathrm{~b}$; BMP, bone morphogenetic protein; BMPr1, BMP receptor 1; BMPr2, BMP receptor 2; R-Smads, receptor-regulated Smads; I-Smads, inhibitory Smads; MAPK, mitogen-activated protein kinase; ERK, extracellular signal-regulated kinase; JNK, c-Jun amino-terminal kinase; mTOR, mammalian target of rapamycin; Bcl2, B-cell lymphoma 2; Mitf, microphthalmia-associated transcription factor; Bim or Bc12L11, Bcl2-like protein 11

\section{Introduction}

In the last decades nucleic acids (NA) are studied as therapeutic tools thanks to interacting at the genetic level, and then generating a specific response. In order to uncover their molecular mechanism, it is important to understand the signaling pathways of the different cellular populations. This work aims to state a clear view of promising NAs and their molecular mechanism in relation to bone-targeted therapy.

Therapeutical NAs can be separated into two groups. One group is formed by plasmid DNA, small interfering RNAs (siRNA), ribozymes, deoxyribozymes, microRNA analogs (miRNA), and microRNA inhibitors (antagomir); which are base pairing complementary to the target. The other group is formed by aptamers, messenger RNAs (mRNA), and $\mathrm{CpG}$; which are not base pairing complementary to the target. NAs are very fragile biomolecules outside the nucleus environment. ${ }^{1}$ Thus, NAs are easily destroyed either in blood, by renal clearance, or hydroxylation by ribozymes and deoxyribozymes. This is why NAs need a special mechanism to be delivered into the cells. Nanoscale biotechnology and tissue engineering have been remarkably important to aim this goal. There is a great variety of nanocarriers for therapeutic NAs that are in preclinical phases and others are already in clinical ones. ${ }^{2-7}$

Understanding the complicated and intricated signaling pathways is a key requirement in order to correctly apply future strategies with NAs. In relation to bone, there is a vast bibliography that compiles the main mechanisms that regulate the balance of bone turnover. In this paper, we present in a summarized way and, trying to be simple, the main metabolic pathways and their responses in relation to cellular differentiation and bone formation.

\section{Signaling pathways in osteoblast/osteocyte lineage}

\section{Wnt pathways}

Wnts are a superfamily of ligands, formed by a chain from 340 to 380 amino acids. Wnts proteins bind to its receptor, a Frizzled protein $(\mathrm{Fz})$ joined to a low-density lipoprotein receptor-related protein 5 or 6 (LRP5/6). Four kinds of signaling pathways assigned to Wntligands have been discovered. One of these signaling pathways is commonly known as the canonical way, while the others are named "non-canonical" ways. This regards the timing of the mechanism chronologically discovered. The non-canonical ways are divided in three trunks; two are activated by the same receptor as the canonical way, but one is activated by a receptor made of Fz joined to RARrelated orphan receptor (ROR). ${ }^{8,9}$

The canonical way depends on $\beta$-catenin. In normal conditions, these proteins join between them, and rapidly, they are destroyed by an APC-Axin-GSK3-CK1 formed complex. This maintains a low concentration of intracellular $\beta$-catenin union. The mechanism is triggered due GSK3, which phosphorylates $\beta$-catenins. Phosphorylated $\beta$-catenins are ubiquitinated and then degraded by proteasome. When Wnts ligands bind toFz and LRP5/6, APC-AxinGSK3-CK1 complex is inhibited, resulting in $\beta$-catenins accumulation in cytosol, and then formation of $\beta$-catenins complex, that triggers 
translocation to the nucleus. $\beta$-catenins complexes regulate specific $\mathrm{T}$ cell factor/lymphoid enhancer factor (TCF/LEF). TCF/LEF regulates Runx2 and Sp7/Osterix, both are the most described and analyzed transcription factors involved in bone formation and differentiation. Both Runx2 and Sp7/Osterix, positively regulates mRNA of proteins like osteocalcin (OCN), osteopontin (OPN), RANK-L, ALP, OPG, Col1, MMP-13, and bone sialoprotein. ${ }^{10-13}$

Wnts are a family ligand. On one hand, the role model of agonism of Wnt family members is Wnt3a. But in another, there is a group of Wnt antagonists like DKK-1, sclerostin, SFRP-1, Wnt5a, and Wnt5b, among others. These proteins are full antagonists of Fz and LRP5/6 receptors. The binding of these proteins to Fz-LRP5/6 regulate by negative feedback on the Wnt pathway. ${ }^{14-17}$ The non-canonical ways like $\mathrm{Ca}^{++}$pathway, PCP pathway and YAP-TAZ pathway are also fundamental for bone formation and differentiation, but we will not discuss them here because the different NAs described in this work interact with participants of the canonical way. We invited to interested and curious readers to expand the reading with the alternative Wnts ways to have a widely panorama of this complex signaling pathways. ${ }^{18-22}$

\section{BMP}

Bone morphogenetic protein (BMP) is growth factors and members of the transforming growth factor $\beta$ (TGF- $\beta$ ). BMP binds to a receptor, that is confirmed by BMP receptor 1 (BMPr1) alone or together with BMP receptor 2 (BMPr2). When BMPs bind to the receptor, this triggers the activation of Smad1, Smad5, and Smad8. These three Smad proteins are known as receptor-regulated Smads (R-Smads). R-Smads binds to Smad4, and then translocase to the nucleus to stimulate Runx2. Smad6 and Smad7, another Smad proteins, inhibit R-Smads complex, and are known as inhibitory Smads (I-Smads). ${ }^{8,23,24}$

\section{ERK}

Mitogen-activated protein kinase (MAPK) controls a great amount of physiological processes. MAPKs activate other kinases, like extracellular signal-regulated kinase (ERK), or c-Jun amino-terminal kinase (JNK). Particularly, ERK activates critical transcription factors like Runx2, and Sp7/Osterix through Sp1, but also E2F, mTOR. mTOR activates Bcl2, and Mitf. Mitf inhibits Bim. This actions triggered by mTOR result in decreased apoptosis and increased survival. ${ }^{25-27}$

In Figure 1, we briefly summarized the main mediators of Wnt, BMP, and ERK signaling pathways, and their principal transcription factors.

\section{Therapeutic nucleic acids and their mechanism}

There are four types of NAs, siRNAs conjugated to $\mathrm{N}$-acetylgalactosamine (GalNac) more specifically, that are in phase III of clinical trials. These drugs are lumasiran, vutrisiran, givosiran, and inclisiran, which are agents against primary hyperoxaluria type 1 , transthyretin mediated amyloidosis, acute hepatic porphyria, and hypercholesterolemia, respectively. ${ }^{1,28-31}$

Like the mentioned siRNAs, there are a huge group of miRNAs and antagomirs that are already identified as remarkably stimulators of osteoblast/osteocytic lineage differentiation, proliferation, and survival. In Table 1, we show the most studied miRNAs and antagomirs in relation to bone signaling pathways, their targets, and their final response to bone turnover. In Figure 1, we show how these NAs interact with aforementioned main signaling pathways.

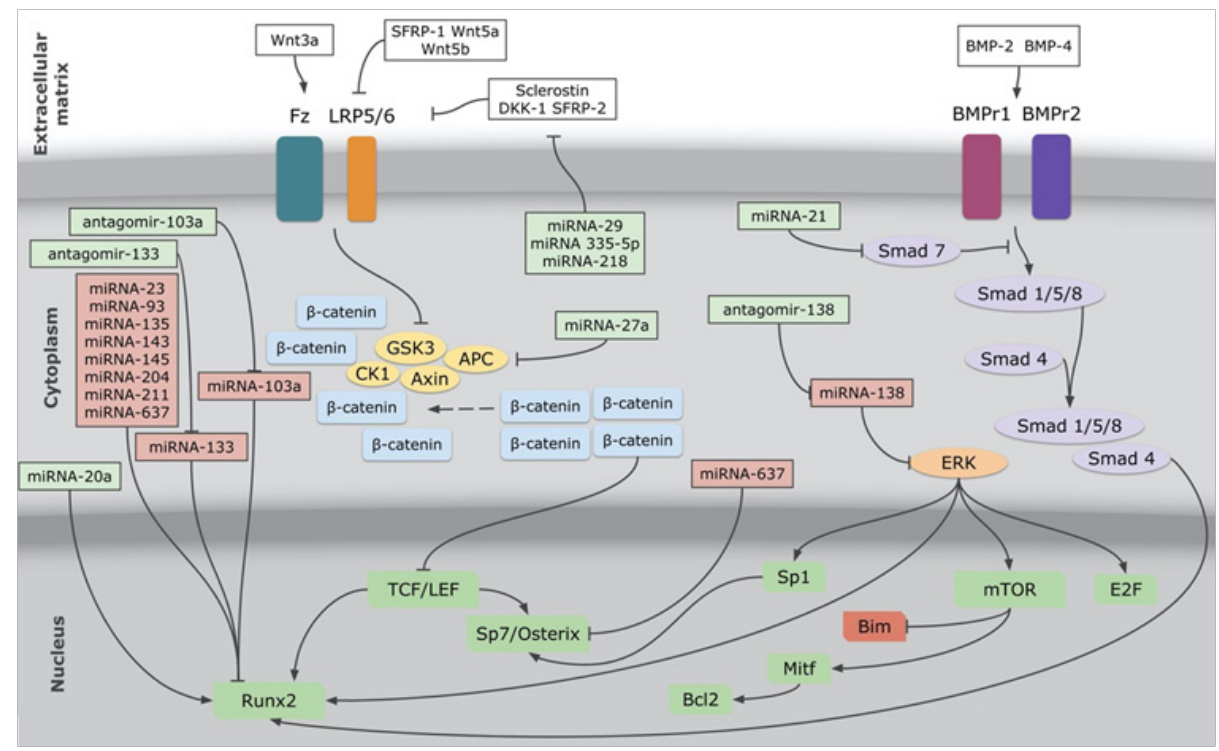

Figure I The figure shows miRNAs and antagomirs, and their interaction with Wnt, BMP and ERK signaling pathways. Connectors with arrows mean stimulation, connectors with flat ending mean inhibition. miRNAs and antagomirs in green boxes mean that they have, as finally response, stimulation of bone formation and differentiation. miRNAs in red boxes mean that they have, as finally response, inhibition of bone formation and differentiation. Transcription factors in green boxes mean that they have, as finally response, stimulation of bone formation and differentiation. Bim in the red box mean that it has, as finally response, inhibition of bone formation.

miRNA, microRNA analog; antagomir, microRNA antagonist; Fz, Frizzled; LRP5/6, low-density lipoprotein receptor-related protein 5 or 6; APC, activated protein C; GSK3, glycogen synthase kinase 3; CKI, casein kinase ;TCFILEF,T cell factor/lymphoid enhancer factor I;Wnt3a, Wnt family member 3a; DKK-I, dikkopf-related protein I; SFRP-I, secreted Frizzled-related protein I;SFRP-2, secreted Frizzled-relatedprotein2; Wnt5a, Wnt family member 5a; Wnt5b, Wnt family member 5b; BMP-2, bone morphogenetic protein 2; BMP-4, bone morphogenetic protein 4; BMPrl, bone morphogenetic protein receptor I; BMPr2, bone morphogenetic protein receptor 2; BMPrI, BMP receptor I; BMPr2, BMP receptor 2;ERK, extracellular signal-regulated kinase; mTOR, mammalian target of rapamycin; Bcl2, B-cell lymphoma 2; Mitf, microphthalmia-associated transcription factor; Bim or Bcl2LI I, Bcl2-like protein II. 
Table I List of microRNA analogs (miRNA) and microRNA antagonists (antagomir), their targets, and their final response in bone turnover

\begin{tabular}{|c|c|c|c|}
\hline $\begin{array}{l}\text { miRNAs and antagomirs }(*=\text { in } \\
\text { vitro and/or in vivo studies) }\end{array}$ & $\begin{array}{l}\text { Targets ( } \underline{\text { underline }}=\text { target } \\
\text { stimulated; italics }=\text { target inhibited) }\end{array}$ & $\begin{array}{l}\text { Stimulate bone } \\
\text { formation and } \\
\text { differentiation }\end{array}$ & $\begin{array}{l}\text { Inhibit bone formation } \\
\text { and differentiation }\end{array}$ \\
\hline miRNA-20a* & $\underline{\text { Runx2 }}$ & $x$ & \\
\hline miRNA-2I* & Smad7 & $x$ & \\
\hline miRNA-23a & Runx2 & & $x$ \\
\hline miRNA-27a & $A P C$ & $x$ & \\
\hline miRNA-29a & Sclerostin, DKK-I, SFRP-2 & $x$ & \\
\hline miRNA-93 & Runx2 & & $x$ \\
\hline miRNA- 103 & Runx2 & & $x$ \\
\hline miRNA-I33a* & Runx2 & & $x$ \\
\hline miRNA-I 35 & Runx2 & & $x$ \\
\hline miRNA-I 43 & Runx2 & & $x$ \\
\hline miRNA-I 45 & Runx2 & & $x$ \\
\hline miRNA-I 38 & ERK & & $x$ \\
\hline miRNA-204 & Runx2 & & $x$ \\
\hline miRNA-2II & Runx2 & & $x$ \\
\hline miRNA-2। 8 & Sclerostin, DKK-I, SFRP-2 & $x$ & \\
\hline miRNA-335-5p* & Sclerostin, DKK-I, SFRP-2 & $x$ & \\
\hline miRNA-637 & Runx2, Sp7/Osterix & & $x$ \\
\hline antagomir-103a & miRNA-I03a & $x$ & \\
\hline antagomir-133a* & miRNA-I33a & $x$ & \\
\hline antagomir-138* & miRNA-I38 & $x$ & \\
\hline
\end{tabular}

miRNA, microRNA analog; antagomir, microRNA antagonist; APC, activated protein C; DKK-I, dikkopf-related protein I; SFRP-2, secreted Frizzled-related protein2; ERK, extracellular signal-regulated kinase.

As we already mentioned, nucleic acid-based therapy has a big amount of challenges. NAs are rapidly degraded by endonucleases. Nucleases are key to protecting us from virus invasion and missense RNA or DNA accumulation. Further, NA molecules have a very rapid renal clearance in blood. It is shown that the half-life of plasmid DNA following IV infusion is closely to 10 minutes. Besides, NAs are negatively charged molecules, so they cannot diffuse across cell membranes. ${ }^{32-35}$ These challenges are being more and more overcome thanks to nanoscale technology. Vectors consist of entrapping NA molecules in suitable nanocarriers with the objective of avoiding the aforementioned barriers. ${ }^{36,37}$ Then are the latest strategies of different vectors for miRNAs and antagomirs against bone diseases used in vitro and/or in vivo studies.

Castaño and cols. al. used collagen-nanohydroxyapatite scaffolds with miRNA-133a, delivered with non-viral particles. They showed that this scaffold enhanced human mesenchymal stem cells through activation of Runx $2 .{ }^{38}$ Nguyen and cols. used polyethylene glycol hydrogels to create a scaffold, with miRNA. They demonstrated that the delivery of miRNA-20a from the hydrogel constructs enhanced the osteogenic differentiation. ${ }^{39}$ Eskildsen and cols. demonstrate that antagomir-138 regulates osteogenic differentiation of human mesenchymal stem cells in vivo ${ }^{40}$, and $\mathrm{Wu}$ and cols. used hydrogels with antagomir-138, demonstrating a significantly enhanced bone regeneration compared to blank group, as long as increased levels of OCN, OPN, and Col1. ${ }^{41}$ Sui and cols. used nanomaterials to deliver miRNA-355-5p using a lipidoid formulation into osteogenic cells for tissue engineering applications. In that paper and in previous studies, this group concluded that miRNA-355-5p interacts with Wnt signaling pathways in regulating bone development and homeostasis. ${ }^{42,43}$ Wang and cols. used a microarc-oxidized titanium surface with miRNA-21loaded hyaluronic acid nanoparticles, and they confirmed that this scaffold promotes osteogenic differentiation on human bone marrow mesenchymal stem cells. ${ }^{44-46}$

There are no clinical trials entries using miRNAs or antagomirs as bone diseases therapy in clinicaltrial.gov to date. However, there is study from Moscow State University started in 2017, that evaluates the safety and efficacy of a gene-activated bone substitute consisting of octacalcium phosphate and plasmid DNA encoding vascular endothelial growth factor for maxillofacial bone regeneration. In this clinical trial were enrolled 20 participants with either congenital or acquired maxillofacial bone defects or alveolar ridge atrophy. All participants received treatment. The safety and efficacy of the implanted bone substitute was evaluated by clinical examination, comprehensive laboratory test, and computer tomography within 6 months after surgery. There is no results yet. ${ }^{47-49}$ 


\section{miRNA as diagnostic biomarkers}

In the latest decades, the number of researches in relation with miRNA, antagomirs increased significantly. Not only as therapeutics, but also as diagnostic biomarkers. miRNA expression patterns specific types of bone diseases, and this can be used as diagnostic tools. Kocijan and cols. identified significant differences in serum levels of circulating miRNAs both in premenopausal women and posmenopausal, even in men. They identified a group of 19 miRNAs that were excellent discriminators of low-traumatic fractures, regardless of age and gender. ${ }^{50}$ Like Kocijan and cols., there are multiple groups studying the changes of different miRNAs levels in serum and/or plasma around the globe, involving different types of population. miRNA-21, miRNA-23a, miRNA-133a, among others miRNAs circulating in serum and/or plasma have a direct correlation with bone mineral density, independent of gender in osteoporotic patients. $^{51-55}$ The European Company TAmiRNAGmbH developed osteomiR $^{\circledR}$, first osteoporotic and high fracture risk diagnostic test based on miRNA in serum or plasma. OsteomiR ${ }^{\circledR}$ - uses qPCR amplification of different types of miRNAs..$^{56}$

Circulating levels of miRNAs were also studied during osteoporosis treatment. Teriparatide and denosumab are two very potent anti-osteoporotic medications. Teriparatide mimics human parathyroid hormone, promoting calcium absorption and bone formation. Denosumab is a monoclonal antibody against RANKL, preventing RANK activation and osteoclast formation. Both drugs were studied in relation to changes in miRNAs expression during treatment. ${ }^{57,58}$ miRNA-133a and miRNA-33 levels were significantly decreased after treatment with teriparatide. ${ }^{51,52,59}$ In relation with denosumab, no significant change of miRNAs levels was observed during treatment. ${ }^{58,60}$

\section{Conclusion}

The knowledge of main signaling pathways is of utmost relevance to understanding the different mechanisms of miRNAs and antagomirs. Noting the difficulties of these molecules in relation to pharmacokinetic is important to generate specific responses using nanotechnology and tissue engineering techniques. Beyond those already mentioned and well-documented in vitro and/or in vivo studies, we are still far from secure, safe, and effective miRNAs and/ or antagomirs-scaffolds for extended use at the clinical level for bone healing in different pathologies. Research into new miRNAs and antagomirs, new delivery strategies and other methods to overcome NAs structural difficulties, and progress towards controlled-clinical studies are key to advancing on bone healing.

\section{Acknowledgments}

None.

\section{Conflicts of interest}

Authors declare that there is no conflict of interest.

\section{References}

1. Weng Y, Huang Q, Li C, et al. Improved Nucleic Acid Therapy with Advanced Nanoscale Biotechnology. Molecular Therapy - Nucleic Acids. 2020;19:581-601.

2. Zhang R, Men K, Zhang X, et al. Delivery of a Modified mRNA Encoding IL-22 Binding Protein (IL-22BP) for Colon Cancer Gene Therapy. J Biomed Nanotechnol. 2018;14(7):1239-1251.

3. Khan OF, Zaia EW, Jhunjhunwala S, et al. Dendrimer-Inspired
Nanomaterials for the in Vivo Delivery of siRNA to Lung Vasculature. Nano Lett. 2015;15(5):3008-3016.

4. Kranz LM, Diken M, Haas H, et al. Systemic RNA delivery to dendritic cells exploits antiviral defence for cancer immunotherapy. Nature. 2016;534(7607):396-401.

5. van Zandwijk N, Pavlakis N, Kao SC, et al. Safety and activity of microRNA-loaded minicells in patients with recurrent malignant pleural mesothelioma: a first-in-man, phase 1, open-label, dose-escalation study. The Lancet Oncology. 2017;18(10):1386-1396.

6. Tangsangasaksri M, Takemoto H, Naito M, et al. siRNA-Loaded Polyion Complex Micelle Decorated with Charge-Conversional Polymer Tuned to Undergo Stepwise Response to Intra-Tumoral and Intra-Endosomal pHs for Exerting Enhanced RNAi Efficacy. Biomacromolecules. 2016;17(1):246-255.

7. Kamerkar S, LeBleu VS, Sugimoto H, et al. Exosomes facilitate therapeutic targeting of oncogenic KRAS in pancreatic cancer. Nature. 2017;546(7659):498-503.

8. Plotkin LI, Bruzzaniti A. Molecular signaling in bone cells: Regulation of cell differentiation and survival. Advances in Protein Chemistry and Structural Biology. 2019;116:237-281.

9. Maeda K, Kobayashi Y, Koide M, et al. The Regulation of Bone Metabolism and Disorders by Wnt Signaling. IJMS. 2019;20(22):5525.

10. Mihara E, Hirai H, Yamamoto H, et al. Active and water-soluble form of lipidated Wnt protein is maintained by a serum glycoprotein afamin $/ \alpha$ albumin. eLife. 2016;5:e11621.

11. Gordon MD, Nusse R. Wnt Signaling: Multiple Pathways, Multiple Receptors, and Multiple Transcription Factors. J Biol Chem. 2006;281(32):22429-22433.

12. Tanaka K, Kitagawa Y, Kadowaki T. Drosophila Segment Polarity Gene Product Porcupine Stimulates the Posttranslational N -Glycosylation of Wingless in the Endoplasmic Reticulum. J Biol Chem. 2002;277(15):12816-12823.

13. Coudreuse D, Korswagen HC. The making of Wnt: new insights into Wnt maturation, sorting and secretion. Development. 2007;134(1):3-12.

14. Bodine PVN, Billiard J, Moran RA, et al. The Wnt antagonist secreted frizzled-related protein-1 controls osteoblast and osteocyte apoptosis. $J$ Cell Biochem. 2005;96(6):1212-1230.

15. Sutherland MK, Geoghegan JC, Yu C, et al. Sclerostin promotes the apoptosis of human osteoblastic cells: a novel regulation of bone formation. Bone. 2004;35(4):828-835.

16. Almeida M, Han L, Bellido T, et al. Wnt Proteins Prevent Apoptosis of Both Uncommitted Osteoblast Progenitors and Differentiated Osteoblasts by $\beta$-Catenin-dependent and -independent Signaling Cascades Involving Src/ERK and Phosphatidylinositol 3-Kinase/AKT. J Biol Chem. 2005;280(50):41342-41351.

17. Baron R, Kneissel M. WNT signaling in bone homeostasis and disease: from human mutations to treatments. Nat Med. 2013;19(2):179-192.

18. Sugimura R, Li L. Noncanonical Wnt signaling in vertebrate development, stem cells, and diseases. Birth Defects Research Part C: Embryo Today: Reviews. 2010;90(4):243-256.

19. Semenov MV, Habas R, MacDonald BT, et al. SnapShot: Noncanonical Wnt Signaling Pathways. Cell. 2007;131(7):1378.e1-1378.e2.

20. Devenport D. The cell biology of planar cell polarity. Journal of Cell Biology. 2014;207(2):171-179.

21. Piccolo S, Dupont S, Cordenonsi M. The Biology of YAP/TAZ: Hippo Signaling and Beyond. Physiological Reviews. 2014;94(4):1287-1312.

22. Kegelman CD, Mason DE, Dawahare JH, et al. Skeletal cell YAP and TAZ combinatorially promote bone development. FASEB $J$. 2018;32(5):2706-2721. 
23. Lowery JW, Rosen V. The BMP Pathway and Its Inhibitors in the Skeleton. Physiological Reviews. 2018;98(4):2431-2452.

24. Katagiri T, Watabe T. Bone Morphogenetic Proteins. Cold Spring Harb Perspect Biol. 2016;8(6):a021899.

25. Kim JM, Yang YS, Park KH, et al. The ERK MAPK Pathway Is Essentia for Skeletal Development and Homeostasis. IJMS. 2019;20(8):1803.

26. Greenblatt MB, Shim JH, Glimcher LH. Mitogen-Activated Protein Kinase Pathways in Osteoblasts. Annu Rev Cell Dev Biol. 2013;29(1):6379.

27. Johnson GL. Mitogen-Activated Protein Kinase Pathways Mediated by ERK, JNK, and p38 Protein Kinases. Science. 2002;298(5600):19111912.

28. Stoekenbroek RM, Kallend D, Wijngaard PL, et al. Inclisiran for the treatment of cardiovascular disease: the ORION clinical development program. Future Cardiology. 2018;14(6):433-442.

29. Kosmas C, Muñoz Estrella A, Sourlas A, et al. Inclisiran: A New Promising Agent in the Management of Hypercholesterolemia. Diseases. 2018;6(3):63.

30. Sardh E, Harper P, Balwani M, et al. Phase 1 Trial of an RNA Interference Therapy for Acute Intermittent Porphyria. $N$ Engl J Med. 2019;380(6):549-558.

31. Janas MM, Zlatev I, Liu J, et al. Safety evaluation of 2'-deoxy-2'-fluoro nucleotides in GalNAc-siRNA conjugates. Nucleic Acids Research. 2019;47(7):3306-3320.

32. Stein JM. The effect of adrenaline and of alpha- and beta-adrenergic blocking agents on ATP concentration and on incorporation of 32Pi into ATP in rat fat cells. Biochem Pharmacol. 1975;24(18):1659-1662.

33. Yin H, Kanasty RL, Eltoukhy AA, et al. Non-viral vectors for genebased therapy. Nat Rev Genet. 2014;15(8):541-555.

34. Worathumrong N, Grimes AJ. The effect of o-salicylate upon pentose phosphate pathway activity in normal and G6PD-deficient red cells. $\mathrm{Br}$ J Haematol. 1975;30(2):225-231.

35. Renaud B, Buda M, Lewis BD, et al. Effects of 5,6-dihydroxytryptamine on tyrosine-hydroxylase activity in central catecholaminergic neurons of the rat. Biochem Pharmacol. 1975;24(18):1739-1742.

36. Wiesmann UN, DiDonato S, Herschkowitz NN. Effect of chloroquine on cultured fibroblasts: release of lysosomal hydrolases and inhibition of their uptake. Biochem Biophys Res Commun. 1975;66(4):1338-1343.

37. Horiike K, Nishina Y, Miyake Y, et al. Affinity labeling of D-amino acid oxidase with an acetylenic substrate. J Biochem. 1975;78(1):57-63.

38. Mencía Castaño I, Curtin CM, Duffy GP, et al. Next generation bone tissue engineering: non-viral miR-133a inhibition using collagennanohydroxyapatite scaffolds rapidly enhances osteogenesis. Sci Rep. 2016;6(1):27941

39. Nguyen MK, Jeon O, Krebs MD, et al. Sustained localized presentation of RNA interfering molecules from in situ forming hydrogels to guide stem cell osteogenic differentiation. Biomaterials. 2014;35(24):62786286

40. Eskildsen T, Taipaleenmaki H, Stenvang J, et al. MicroRNA-138 regulates osteogenic differentiation of human stromal (mesenchymal) stem cells in vivo. Proceedings of the National Academy of Sciences. 2011;108(15):6139-6144.

41. Wu G, Feng C, Quan J, et al. In situ controlled release of stromal cell-derived factor- $1 \alpha$ and antimiR-138 for on-demand cranial bone regeneration. Carbohydrate Polymers. 2018;182:215-224.

42. Sui L, Wang M, Han Q, et al. A novel Lipidoid-MicroRNA formulation promotes calvarial bone regeneration. Biomaterials. 2018;177:88-97.
43. Zhang L, Tang Y, Zhu X, et al. Overexpression of MiR-335-5p Promotes Bone Formation and Regeneration in Mice: in vivo effects of MiR-335-5p on bone formation and regeneration. $J$ Bone Miner Res. 2017;32(12):2466-2475.

44. Zhao Y, Wang $\mathrm{Z}$, Wu G, et al. Microarc-oxidized titanium surfaces functionalized with microRNA-21-loaded chitosan/hyaluronic acid nanoparticles promote the osteogenic differentiation of human bone marrow mesenchymal stem cells. IJN. 2015;10:6675-6687.

45. Zhao Y, Wang Z, Wu G, et al. Improving the osteogenesis of human bone marrow mesenchymal stem cell sheets by microRNA-21-loaded chitosan/hyaluronic acid nanoparticles via reverse transfection. IJN 2016;11:2091-2105.

46. Leng Q, Chen L, Lv Y. RNA-based scaffolds for bone regeneration: application and mechanisms of mRNA, miRNA and siRNA. Theranostics. 2020;10(7):3190-3205.

47. Bozo IY, Deev RV, Drobyshev AY, et al. World's First Clinical Case of Gene-Activated Bone Substitute Application. Case Reports in Dentistry. 2016;2016:1-6.

48. Deev RV, Drobyshev AY, Bozo IY, et al. Ordinary and Activated Bone Grafts: Applied Classification and the Main Features. BioMed Research International. 2015;2015:1-19.

49. https://clinicaltrials.gov/ct2/show/NCT03076138.

50. Kocijan R, Muschitz C, Geiger E, et al. Circulating microRNA Signatures in Patients With Idiopathic and Postmenopausal Osteoporosis and Fragility Fractures. The Journal of Clinical Endocrinology \& Metabolism. 2016;101(11):4125-4134.

51. Li H, Wang Z, Fu Q, Zhang J. Plasma miRNA levels correlate with sensitivity to bone mineral density in postmenopausal osteoporosis patients. Biomarkers. 2014;19(7):553-556.

52. Wang Y, Li L, Moore BT, et al. MiR-133a in Human Circulating Monocytes: A Potential Biomarker Associated with Postmenopausal Osteoporosis. PLoS ONE. 2012;7(4):e34641.

53. Seeliger C, Karpinski K, Haug AT, et al. Five Freely Circulating miRNAs and Bone Tissue miRNAs Are Associated With Osteoporotic Fractures: miRNAs associated with osteoporotic fractures. J Bone Miner Res. 2014;29(8):1718-1728.

54. Kelch S, Balmayor ER, Seeliger C, et al. miRNAs in bone tissue correlate to bone mineral density and circulating miRNAs are gender independent in osteoporotic patients. Sci Rep. 2017;7(1):15861.

55. Yavropoulou MP, Anastasilakis AD, Makras P, et al. Expression of microRNAs that regulate bone turnover in the serum of postmenopausal women with low bone mass and vertebral fractures. European Journal of Endocrinology. 2017;176(2):169-176.

56. Walter E, Dellago H, Grillari J, et al. Cost-utility analysis of fracture risk assessment using microRNAs compared with standard tools and no monitoring in the Austrian female population. Bone. 2018;108:44-54.

57. Anastasilakis AD, Makras P, Pikilidou M, et al. Changes of Circulating MicroRNAs in Response to Treatment With Teriparatide or Denosumab in Postmenopausal Osteoporosis. The Journal of Clinical Endocrinology \& Metabolism. 2018;103(3):1206-1213.

58. Anastasilakis AD, Yavropoulou MP, Makras P, et al. Increased osteoclastogenesis in patients with vertebral fractures following discontinuation of denosumab treatment. European Journal of Endocrinology. 2017;176(6):677-683.

59. Cheng VKF, Au PCM, Tan KC, et al. MicroRNA and Human Bone Health: microrna and human bone health. JBMR Plus. 2019;3(1):2-13.

60. Zhang W, Wu Y, Shiozaki Y, etal. miRNA-133a-5p Inhibits the Expression of Osteoblast Differentiation-Associated Markers by Targeting the 3 UTR of RUNX2. DNA and Cell Biology. 2018;37(3):199-209. 